\title{
Sulphur XANES Analysis of Cultured Human Prostate Cancer Cells
}

\author{
W.M. Kwiatek ${ }^{a, *}$, M. PodgórczyK $^{a}$, Cz. Paluszkiewicz $^{b}$, \\ A. BALERNA ${ }^{c}$ AND A. KISIEL ${ }^{d}$ \\ ${ }^{a}$ The Henryk Niewodniczański Institute of Nuclear Physics \\ Polish Academy of Sciences, Radzikowskiego 152, 31-342 Cracow Poland \\ ${ }^{b}$ AGH University of Science and Technology \\ al. Mickiewicza 30, 30-059 Cracow, Poland \\ ${ }^{c}$ INFN - Laboratori Nazionali di Frascati \\ Via E. Fermi 40, I-00044 Frascati, Italy \\ ${ }^{d}$ Institute of Physics, Jagiellonian University \\ Reymonta 4, 30-059 Cracow, Poland
}

\begin{abstract}
Prostate cancer is one of the most commonly diagnosed cancers in men throughout the world. It is believed that changes to the structure of protein binding sites, altering its metabolism, may play an important role in carcinogenesis. Sulphur, often present in binding sites, can influence such changes through its chemical speciation. Hence there is a need for precise investigation of coordination environment of sulphur. X-ray absorption near edge structure spectroscopy offers such possibility. Cell culture samples offer histologically well defined areas of good homogeneity, suitable for successful and reliable X-ray absorption near edge structure analysis. This paper presents sulphur speciation data collected from three different human prostate cancer cell lines (PC-3, LNCaP and DU-145). Sulphur X-ray absorption near edge structure analysis was performed on $K$-edge structure. The spectra of cells were compared with those of cancerous tissue and with organic substances as well as inorganic compounds.
\end{abstract}

PACS numbers: 87.64.kd, 87.19.xj

\section{Introduction}

Sulphur is the eighth most abundant element to be found in human body. For example, it contributes to the structure of amino acids such as cysteine and cystine, and hence it may affect protein functionality. The latter becomes an issue whenever

*corresponding author; e-mail: wojciech.kwiatek@ifj.edu.pl 
sulphur enters protein binding sites, as then the influence of this element upon the activity of proteins becomes direct. Any changes in protein structure (especially in their active parts such as binding sites) can therefore alter the protein activity and, consequently, affect metabolism of a cell and tissue. Such changes are believed to be very first steps of still poorly understood process of carcinogenesis. This makes the investigations of sulphur surroundings in biomolecules an important task on the way to understanding pathogenesis of cancer.

There are very few tools for studying the role of sulphur in biological systems, and among them X-ray absorption near edge structure (XANES) spectroscopy offers a unique non-destructing possibility for determining oxidation state of the element and characterising its chemical speciation $[1,2]$.

Our special interest was focused on human prostate cancer due to its prevalence among diagnosed cancers in men [3] and driven by the need of understanding its aetiology.

The choice of sulphur as a probe element in this work is a consequence of its key role in prostate cell metabolism $[4,5]$. Sulphur ligands provide a redox-active coordination environment for the redox-inert zinc ions, present in binding sites of proteins involved in many important processes such as DNA recognition, RNA packaging, activation of transcription process, regulation of apoptosis and lipid binding.

The main aim of this study was to analyse the structure of XANES spectra of human prostate cancer cell lines and to compare them with the spectra taken on prostate cancer tissue and reference organic compounds. Such comparison could be a first step in search for the chemical phenomena associated with carcinogenesis.

XANES analysis of biological samples, especially of a tissue, is by no means straightforward since the experimental beam may illuminate structures of different pathological status, still remaining histologically indistinguishable due to the early stage of pathogenesis. It is therefore crucial for the reliability of the results that experimental information be collected from homogeneous samples. Required homogeneity can be assured by choosing cell samples that are a priori well defined histologically. For this reason, and on the grounds of our previous analysis performed on tissue samples [6], cultured human prostate cancer cell lines were used in this study, along with human cancerous tissue material.

\section{Sample preparation}

Three human prostate cancer cell lines, named LNCaP, DU-145 and PC3, were cultured at the Department of Applied Spectroscopy, Institute of $\mathrm{Nu}-$ clear Physics PAN and the Chair of Medical Biochemistry, Jagiellonian University Medical College, Kraków. Cells were grown in $25 \mathrm{~cm}^{2}$ culture dishes using RPMI-1640 medium (SIGMA-Aldrich) supplemented with 10\% fetal bovine serum (SIGMA-Aldrich), $100 \mathrm{U} / \mathrm{ml}$ penicillin-streptomycin solution (SIGMA-Aldrich), $10 \mathrm{mM}$ HEPES (SIGMA-Aldrich), $1 \mathrm{mM}$ sodium pyruvate (SIGMA-Aldrich) and 
$4.5 \mathrm{~g} / 1$ glucose. Culture dishes were maintained at $37^{\circ} \mathrm{C}$ in a humidified atmosphere of $5 \% \mathrm{CO}_{2}$ and the culture medium was replaced every $72 \mathrm{~h}$. Cells were collected using $0.25 \%$ trypsin in phosphate buffered saline (PBS) (SIGMA-Aldrich) and subsequently centrifuged at $1800 \mathrm{rpm}$ for $4 \mathrm{~min}$. In order to fix cells, a $2 \%$ paraformaldehyde in PBS solution or $2 \%$ glutharaldehyde in PBS solution were used. Cells were kept in the above solutions for $30 \mathrm{~min}$ in room temperature and then for $2 \mathrm{~h}$ at $4^{\circ} \mathrm{C}$. Upon completion of fixation, two $50 \mu \mathrm{l}$ drops of cells suspension were placed on $1.5 \mu \mathrm{m}$ thick mylar foil (Goodfellow) and let to dry. For reference, samples of unfixed cells (only suspended in PBS) were prepared as well.

Prostate tissue samples, obtained during surgery on patients suffering from prostate cancer, were cut into $14 \mu \mathrm{m}$ thick slices with a cryomicrotome, and placed on $1.5 \mu \mathrm{m}$ thick mylar foil (Goodfellow). One section of each sample was used for XANES analysis, while another was stained with hematoxylin and eosin for histological examination.

\section{Methods}

Sulphur K-edge XANES experiments on cell lines and tissue samples were performed at the DRX1 beamline at LNF, Frascati (Italy). Soft X-ray synchrotron radiation coming out of a DAFNE wiggler magnet was let through a Toyama double-crystal monochromator to achieve the energy ranging from $1200 \mathrm{eV}$ to $3100 \mathrm{eV}$, depending on the crystal pair chosen. Such radiation is well suited for XANES measurements on $K$-edges of light elements starting from $\mathrm{Mg}$ onwards, and on $L$-edges and $M$-edges of elements with higher atomic numbers.

The experiment was carried out in transmission mode, at room temperature, in vacuum, using a broad beam of $1 \mathrm{~mm} \times 8 \mathrm{~mm}$ section. During measurements the energy was scanned from $2460 \mathrm{eV}$ to $2510 \mathrm{eV}$ with the step of $0.2 \mathrm{eV}$. The acquisition time for each energy point was set to $1 \mathrm{~s}$ for standards and to $2 \mathrm{~s}$ for tissue and cells samples. In order to find oxidation state of sulphur present in samples, reference inorganic compounds: $\mathrm{S} 8, \mathrm{ZnS}, \mathrm{CdS}, \mathrm{K}_{2} \mathrm{~S}_{2} \mathrm{O}_{5}, \mathrm{MgSO}_{4}, \mathrm{Na}_{2} \mathrm{SO}_{4},\left(\mathrm{NH}_{4}\right)_{2} \mathrm{SO}_{4}$, $\mathrm{BaSO}_{4}$, and $\mathrm{Na}_{2} \mathrm{~S}_{2} \mathrm{O}_{3}$ were chosen for energy calibration of the spectra. Additionally, organic standards: albumin, ovo-albumin, myoglobin, L-cysteine, and H-8 horse kidney (IAEA standard reference material) were measured in the same experimental conditions.

\section{Results}

The collected sulphur $K$-edge XANES spectra were analysed using IFFEFIT software [7]. It included pre-edge and post-edge normalisation as well as background subtraction.

The XANES sulphur $K$-edge spectra of reference inorganic compounds are shown in Fig. 1. They are in good agreement with the previously obtained results by Pickering et al. [2], so the experimental setup was reliably calibrated. 


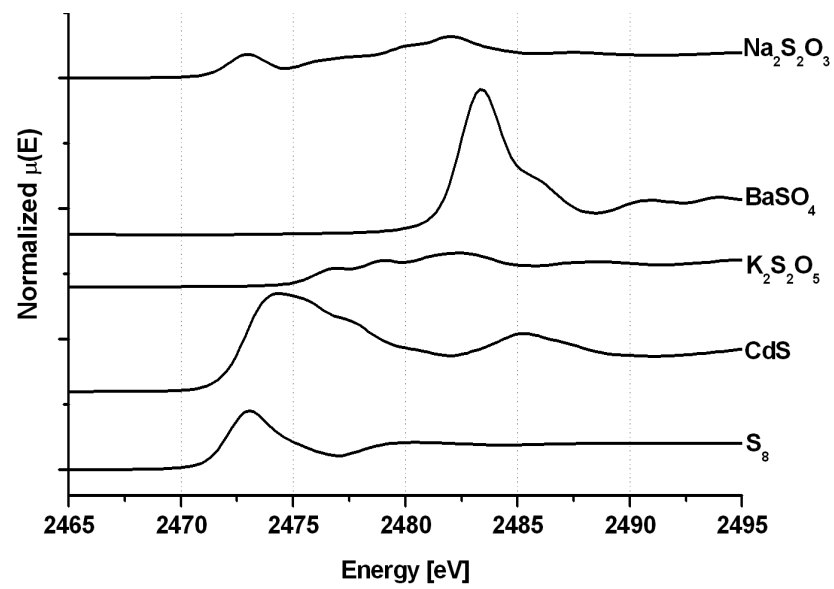

Fig. 1. XANES sulphur $K$-edge spectra of reference inorganic compounds.

It is not enough to analyse spectra of well-known inorganic compounds in order to determine precisely the oxidation state of sulphur in cells, since inorganic crystalline compounds have simpler and better defined structures than those found in living cells. There, looking at sulphur indeed means looking at proteins that contain sulphur. For that reason we analysed XANES spectra of another set of reference material, this time there were proteins and amino acids.

S peak positions and crystallographic structures [8] of inorganic compounds.

\begin{tabular}{|c|c|c|c|c|}
\hline Compound & $\begin{array}{l}\text { S oxidation } \\
\text { state }\end{array}$ & $\begin{array}{l}\text { White peak } \\
\text { position }[\mathrm{eV}]\end{array}$ & Structure & $\begin{array}{l}\text { Space } \\
\text { group }\end{array}$ \\
\hline $\mathrm{S}_{8}$ & 0 & $2473,8 \pm 0.2$ & monoclinic-beta & $P 2 / c$ \\
\hline $\mathrm{ZnS}$ & $-\mathrm{II}$ & $2475.0 \pm 0.2$ & cubic & $F-43 m$ \\
\hline CdS & $-\mathrm{II}$ & $2474.2 \pm 0.2$ & hexagonal & $P 63 m c$ \\
\hline $\mathrm{K}_{2} \mathrm{~S}_{2} \mathrm{O}_{5}$ & $+\mathrm{IV}$ & $2482.5 \pm 0.2$ & monoclinic & $P 121 / m 1$ \\
\hline $\mathrm{MgSO}_{4}$ & $+\mathrm{VI}$ & $2483.9 \pm 0.2$ & orthorhombic & Cmcm \\
\hline$\left(\mathrm{NH}_{4}\right)_{2} \mathrm{SO}_{4}$ & $+\mathrm{VI}$ & $2483.5 \pm 0.2$ & orthorhombic & Pna21 \\
\hline $\mathrm{BaSO}_{4}$ & $+\mathrm{VI}$ & $2483.3 \pm 0.2$ & orthorhombic & Pnma \\
\hline $\mathrm{Na}_{2} \mathrm{~S}_{2} \mathrm{O}_{3}$ & $-\mathrm{II}$ and $+\mathrm{VI}$ & $\begin{array}{l}2473.0 \pm 0.2 \\
2481.9 \pm 0.2\end{array}$ & monoclinic & $P 121 / a 1$ \\
\hline
\end{tabular}

*the energy step of $0.2 \mathrm{eV}$ was assumed as an estimated error of white peak position

The position of white peaks of sulphur $K$-edge XANES spectra for inorganic standards are presented in Table, along with sulphur oxidation state and crystallographic data.

The white peak position appears to depend on both $\mathrm{S}$ oxidation state and crystallographic structure of the compound. Shifts of $K$-edge and white peak po- 
sitions in $\mathrm{ZnS}$ and $\mathrm{CdS}$ with respect to $\mathrm{S}_{8}$ differ, as a result of different structures. Although $\mathrm{MgSO}_{4},\left(\mathrm{NH}_{4}\right)_{2} \mathrm{SO}_{4}$, and $\mathrm{BaSO}_{4}$ share the same crystal system, their structures belong to different space groups. Even though sulphur oxidation states are the same, slight differences in both $K$-edge and white peak positions can result from the compounds not being isostructural. Interesting edge features reveals the $\mathrm{Na}_{2} \mathrm{~S}_{2} \mathrm{O}_{3}$ XANES spectrum, whose two maxima indicate the presence of two sulphur atoms in energy-inequivalent positions.

The S $K$-edge XANES spectra for cell samples were analysed in the same manner as for standards. In order to check the possible influence of cell fixing method upon XANES, spectra of the LNCaP cell line were measured thrice: (i) fixed with $2 \%$ paraformaldehyde in PBS solution, (ii) fixed with $2 \%$ glutharaldehyde in PBS solution, and (iii) not fixed but suspended in PBS (see Fig. 2). The observed energy peaks around $2474 \mathrm{eV}$ and $2482 \mathrm{eV}$ practically coincide, thus disproving any possible dependence of their position upon the fixing procedure. Moreover, location of those peaks could be associated with the presence of sulphur (-II) and sulphur (+VI), and is very similar to what is observed in $\mathrm{Na}_{2} \mathrm{~S}_{2} \mathrm{O}_{3}$ (see Fig. 1). This is by no means a surprise: a broad beam produces XANES signal that is averaged over a large sample area, containing a wealth of sulphur-containing molecules.

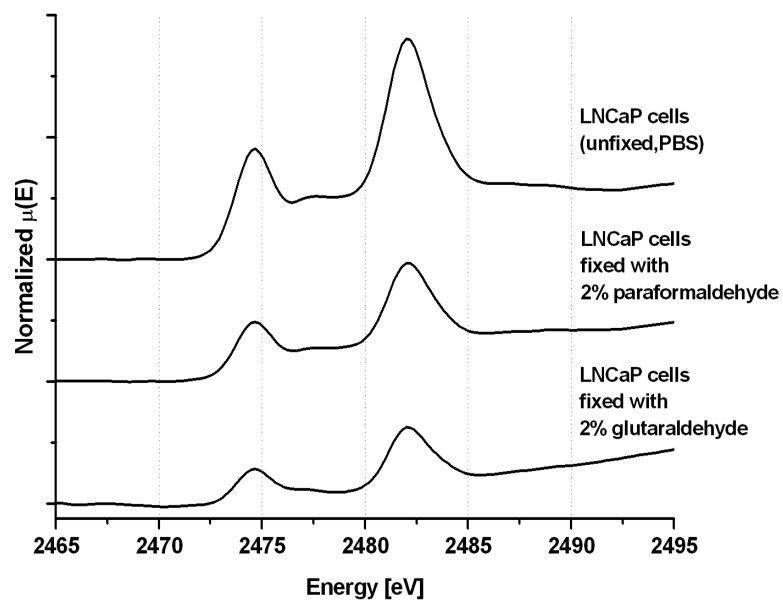

Fig. 2. Sulphur $K$-edge XANES spectra of LNCaP cell line: not fixed but suspended in PBS, fixed with $2 \%$ paraformaldehyde in PBS solution, and fixed with $2 \%$ gluteraldehyde in PBS solution.

Another problem consists in detecting and explaining possible differences in XANES spectra among three human prostate cancer cell lines and cancer tissue. Figure 3 compares such spectra measured for cell lines fixed with $2 \%$ paraformaldehyde. The peaks at about $2482 \mathrm{eV}$ are higher than those at about $2474 \mathrm{eV}$, suggesting higher concentration of sulphur (+VI) than of sulphur (-II) in the samples. 
Contrary to this characteristic of cultured cell lines, no such intensity difference between $2482 \mathrm{eV}$ and $2474 \mathrm{eV}$ peaks exists in the spectra of prostate cancer tissue. Moreover, the latter sample gives a pronounced third peak around $2477 \mathrm{eV}$, almost absent in cultured cell lines. This maximum can be attributed to sulphur $(+\mathrm{IV})$.

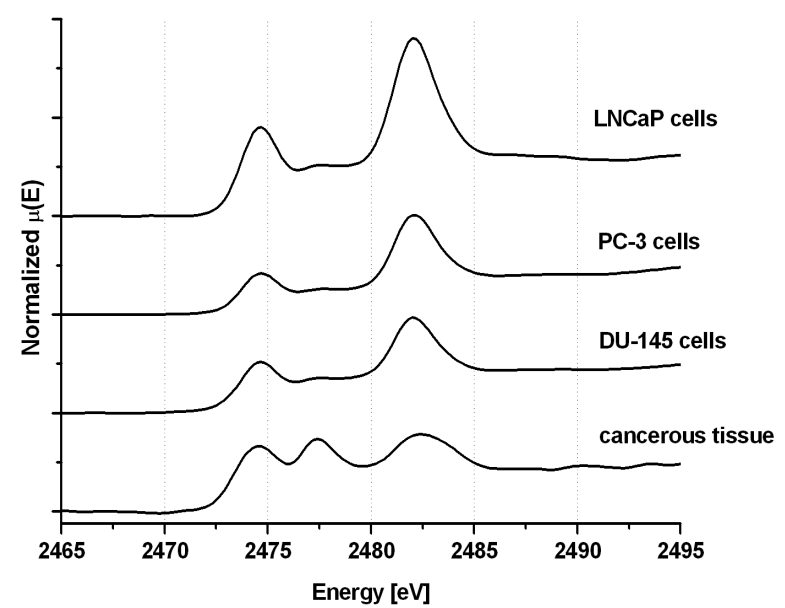

Fig. 3. Sulphur $K$-edge XANES spectra of different prostate cancer cell lines and prostate cancer tissue.

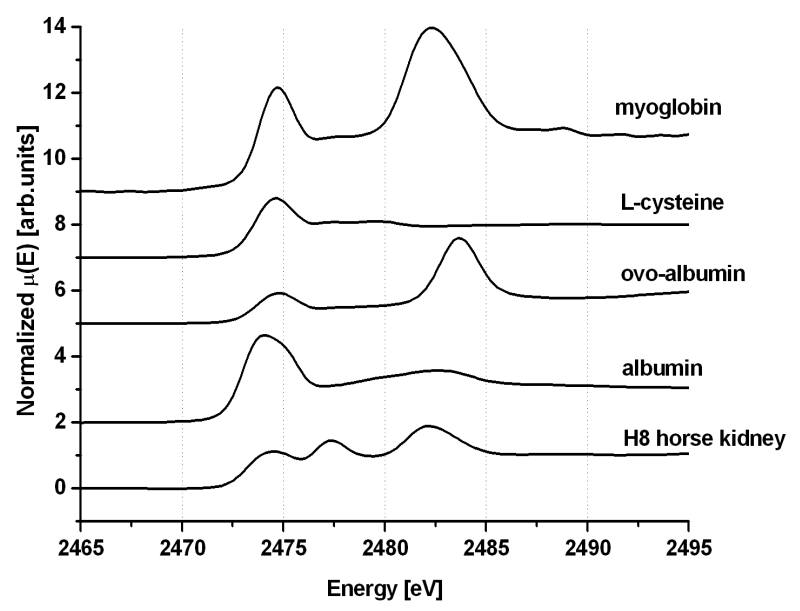

Fig. 4. Sulphur $K$-edge XANES spectra of reference organic compounds.

Cultured cells are single-type, while cancerous tissue contains a variety of cells, and certain damage to the protein structure in pathological cells, resulting in a change of sulphur coordination, affecting its oxidation state, cannot be ruled out. Nevertheless, the origin of the third peak requires further study. 
The above mentioned organic reference samples were measured under conditions identical to those applied for the cancer cells under study. The spectra obtained in this way are presented in Fig. 4. Myoglobin and ovo-albumin yield bimodal XANES signals of comparable intensity ratio between peaks. Moreover, the higher-energy one in ovo-albumin is slightly shifted with respect to its myoglobin counterpart. Peak of dominant intensity is observed for albumin and L-cysteine at $c a .2474 \mathrm{eV}$. H-8 horse kidney spectrum resembles that of prostate cancer tissue with respect to positions on the energy scale (Fig. 3) but not with respect to intensities. Again, the spectrum of a tissue standard sample differs from that of cells as a result of averaging experimental technique, taking contribution from all proteins present both inside cells and outside - in the extracellular space.

\section{Conclusion}

Sulphur $K$-edge position for cultured cell lines does not depend on fixing procedure. For different cultured prostate cancer cell lines (PC-3, DU-145, LNCaP) and prostate cancer tissue the XANES spectra have peaks located at about $2474 \mathrm{eV}, 2477 \mathrm{eV}, 2482 \mathrm{eV}$, and $2498 \mathrm{eV}$ in the Kossel region. Comparison of obtained cells spectra with those of inorganic standards shows similarity in both peak positions and their intensity ratio to the $\mathrm{Na}_{2} \mathrm{~S}_{2} \mathrm{O}_{3}$ spectrum, where sulphur exists in $(-\mathrm{II})$ and $(+\mathrm{VI})$ oxidation states. An interesting intensity inversion of peaks at $c a .2474 \mathrm{eV}$ and $2482 \mathrm{eV}$ in organic samples with respect to $\mathrm{Na}_{2} \mathrm{~S}_{2} \mathrm{O}_{3}$ is observed which may indicate different proportion of sulphur in $(-\mathrm{II})$ and $(+\mathrm{VI})$ oxidation states. Spectrum of prostate cancer tissue is comparable with that of H-8 horse kidney, whereas cultured cells produce spectra similar to myoglobin and ovo-albumin. Pronounced peaks at ca. $2477 \mathrm{eV}$ in tissue and standard H- 8 spectra may indicate the presence of sulphur $(+\mathrm{IV})$, almost absent in the spectra of cultured cells. Several topics, including the latter, require further study. Density of states calculation [9] may shed more light on these issues.

\section{Acknowledgments}

The authors wish to thank Prof. Jerzy Stachura and Prof. Tadeusz Cichocki from Collegium Medicum, Jagiellonian University for their assistance in tissue samples classification and preparation as well as to Prof. Piotr M. Laidler and to Dr. Joanna Dulińska-Litewka for cultured prostate cells samples preparation, and to dr. Marek Gałka from the Gabriel Narutowicz Hospital in Kraków for sample procurement. Special thanks are due to Dr. Wojciech Zając from the Institute of Nuclear Physics PAN for fruitful discussions and help in revision of the manuscript. This work was supported by EU Integrated Infrastructure Initiative Hadron Physics Project, contract No. RII3-CT-2004-506078, Tari Program No. 48 and bz MNiSW as a research project, grant N0. WN 30146734. 


\section{References}

[1] A. Vairavamurthy, Spectrochim. Acta A 54, 2009 (1998).

[2] I.J. Pickering, R.C. Prince, T. Divers, G.N. George, Federation European Biochemical Societes Lett. 441, 11 (1998).

[3] P. Boyle, G. Severi, G.G. Giles, Urol. Clin. North Am. 30, 209 (2003).

[4] W. Maret, J. Trace Elem. Med. Biol. 19, 7 (2005).

[5] W. Maret, Biochemistry 43, 3301 (2004).

[6] W.M. Kwiatek, A. Banaś, A. Kisiel, G. Cinque, Acta Phys. Pol. A 109, 383 (2006).

[7] B. Ravel, M. Newville, J. Synchrotron Rad. 12, 537 (2005).

[8] Inorganic Crystal Structure Database (ICSD): http://icsdweb.fiz-karlsruhe.de/.

[9] A. Kisiel, G. Dalba, P. Fornasini, M. Podgórny, J. Oleszkiewicz, F. Rocca, E. Burattini, Phys. Rev. B 39, 7895 (1989). 